\section{Complex karyotype with novel translocation in pure erythroid leukemia patient}

\author{
Mansour Aljabry \\ Department of Pathology, King Khalid \\ University Hospital, King Saud \\ University, Riyadh, Saudi Arabia
}

\section{Abstract}

Pure erythroid leukemia (PEL) is rare hematopoietic neoplasm characterized by uncontrolled proliferation of immature erythroid precursors - mainly abnormal proerythroblasts - comprising at least $80 \%$ of bone marrow cells. In this paper, I present a case of 48 years old patient, who presented with pancytopenia and circulating erythroblast in peripheral blood after long history of alcohol abuse. Bone marrow examination revealed hypercellular marrow which is markedly infiltrated with immature erythroid precursors. An expanded panel of immunophenotyping markers has confirmed the diagnosis of PEL. Cytogenetics analysis detected a complex karyotype with multiple chromosomal abnormalities and a novel translocation, $\mathrm{t}(8 ; 9)$ (p11.2;q12), which has not been reported in acute myeloid leukemia (AML) in the past. The patient was treated with standard AML chemotherapy but he did not show an optimal response and passed away. An updated and short review about various aspects of PEL has been made with special focus on immunophenotyping and genetic studies.

\section{Case Report}

\section{Clinical history}

A 48 years old Saudi male presented to the emergency department with shortness of breath, persistent low grade fever and easy bruising for 3 days. The patient also complained of progressive weakness, loss of appetite for 2 months duration and had lost $7 \mathrm{~kg}$ of weight over the last 2 months. His family or medical history was unremarkable and was not receiving any medical treatment for any chronic illness. Social history revealed that he was unemployed with low economic status. He was a heavy smoker consuming two packs of cigarettes daily for several years and had long history of heavy alcohol abuse. He denied any drug abuse or toxin exposure. On physical examination; he was pale, had multiple bruises particularly on the lower limbs and his body temperature was $38.2^{\circ} \mathrm{C}$. There was mild hepatosplenomegaly with no lymphadenopathy.

\section{Methods and investigations}

Hematological investigation revealed hemoglobin of $51 \mathrm{~g} / \mathrm{L}(120-160 \mathrm{~g} / \mathrm{L})$, red blood cells (RBC) $2.15 \times 10^{12} / \mathrm{L}\left(4.2-5.5 \times 10^{12} / \mathrm{L}\right)$, mean corpuscular volume $88 \mathrm{fl}$ (78-97 fl) and mean corpuscular hemoglobin $28 \mathrm{pg}$ (27-32 pg). Leukocytes were $2.01 \times 10^{9} / \mathrm{L}$ with $3 \%$ blasts, myelocytes $3 \%$, neutrophils $19 \%$, monocytes $8 \%$, basophils $1 \%$ and $67 \%$ lymphocytes. Platelet count was $23 \times 10^{9} / \mathrm{L}\left(140-450 \times 10^{9} / \mathrm{L}\right)$. Biochemical markers revealed elevated serum levels of lactate dehydrogenase (LDH), uric acid and total bilirubin. Renal function and liver function tests were within normal limits. Peripheral smear showed marked anisopoikilocytosis with schistocytes, basophilic stippling, spherocytes, many nucleated RBC and rare circulating blasts. Erythroid precursors showed significant dysplastic changes with nuclear irregularities, budding and bi-nucleation while neutrophil morphology was essentially normal. The circulating blasts were medium to large blasts with round nuclei, fine chromatin, prominent nucleoli and deep blue a granular cytoplasm without Auer rods.

The bone marrow aspirate yielded a markedly hypercellular bone marrow essentially replaced by erythroid precursors, representing approximately $87 \%$ of the marrow cells. Of this, the proerythroblasts comprised $58 \%$. The erythroid precursors displayed dysplastic morphology, including multi-nucleation, nuclear budding and megaloblastoid changes. Myeloblasts were about $3 \%$ and megakaryocytes were reduced without obvious dysplasia. Periodic Acid Schiff (PAS) stain revealed globular and block-like positivity in many abnormal erythroblasts. Perls Perl's stain showed a few sideroblasts; however no ringed sideroblasts were observed.

Flow cytometry analysis of the bone marrow aspirate revealed many immature cells which were positive for CD71, glycophorin A and partially positive for CD36, consistent with erythroid precursors. Staining for CD13, CD33, CD34, CD41, CD61 and all other myeloid and lymphoid makers was negative. Myeloblasts were not detected. The bone marrow biopsy revealed a hypercellular packed marrow with monotonous leukemic infiltrate composed of primitive looking blasts. The infiltrating blasts had scant cytoplasm, high nuclear to cytoplasmic ratio, round nucleus with fine chromatin and 1-2 prominent nucleoli (Figure 1).

Immunohistochemistry revealed strong positivity to E-cadherin (ECH-6; Ventana Medical Systems, Inc., Roche, Basel, Switzerland), weak positivity for glycophorin A (GA-R2; Ventana Medical Systems, Inc.) and negative for CD34, MP0, vWF, CD3 and CD79a. These morphological and immunophenotypic findings supported the diagnosis of pure erythroid leukemia (PEL) FAB subtype M6b.
Correspondence: Mansour Semar Aljabry, Hematology Unit, Pathology Department, King Khalid University Hospital, King Saud University, P.0 Box 2925, Riyadh 11461, Kingdom of Saudi Arabia.

Tel.: +966.114.671812

E-mail: m.aljabry4210@gmail.com

Key words: pure erythroid leukemia, acute erythroid leukemia, acute myeloid leukemia.

Conflict of interest: the authors declare no potential conflict of interest.

Funding: this paper has been funded by King Saud University, Deanship of Scientific Research, College of Medicine, Research Center.

Received for publication: 14 October 2014.

Revision received: 20 February 2015.

Accepted for publication: 24 February 2015.

This work is licensed under a Creative Commons Attribution NonCommercial 3.0 License (CC BYNC 3.0).

(C) Copyright M. Aljabry, 2015

Licensee PAGEPress, Italy

Hematology Reports 2015; 7:5674

doi:10.4081/hr.2015.5674

Cytogenetics analysis was performed on 20 metaphases and detected a complex karyotype with multiple chromosomal aberrations (Figure 2):

$$
\begin{gathered}
\text { 46-48,XY,add(4)(q?) } \\
\text { [11]del(4)(p?)[17],del(5)(q23)[8], add(6)(p } \\
\text { 23)[16],del(7)(q22)[12], } \\
\mathrm{t}(8 ; 9)(\mathrm{p} 11.2 ; \mathrm{q} 12)[20],+\operatorname{del}(9)(\mathrm{q} 22)[17], \mathrm{de} \\
\mathrm{l}(18)(\mathrm{q} 21)[13]
\end{gathered}
$$

\section{Hospital course}

The patient was treated with a standard induction $(7+3)$ chemotherapy regimen consisting of daunorubicin $45 \mathrm{mg} / \mathrm{m}^{2}$ daily for 3 days and cytarabine $100 \mathrm{mg} / \mathrm{m}^{2}$ daily continuous infusion for 7 days. Blood and platelet transfusions were administered during induction period. On post-induction day 28 , the bone marrow biopsy showed hypercellular marrow with $38 \%$ residual erythroblasts. Cytogenetics showed persistent multiple chromosomal abnormalities. He was re-inducted again with FLAG regimen (fludarabine + high-dose cytarabine + G-CSF). He failed to tolerate the treatment and died after 10 days due to severe pulmonary infection.

\section{Discussion}

Pure erythroid leukemia is exceedingly rare type of AML representing < $1 \%$ of all AML cases and $13 \%$ of acute erythroid leukemia cases. ${ }^{1}$ It 
is a neoplastic proliferation of immature cells committed exclusively to the erythroid lineage comprising at least $80 \%$ of bone marrow cells with no evidence of a significant myeloblastic component. ${ }^{2}$

Clinically, PEL patients present usually with profound anemia symptoms such as weakness and pallor, fever and hemorrhages. ${ }^{3}$ Presence of hepatosplenomegaly varies between 20 to $40 \%$ of the series. ${ }^{3,4}$ There are few reported cases of extramedullary pure erythroid leukemia involving lymph nodes or presenting as a hemangioma. ${ }^{5,6}$

Our patient presented with PEL post heavy alcohol abuse over a prolonged time period that may indicate presence of some kind of causal relationship, an observation consistent with previous reports. ${ }^{7,8}$

Because of the extreme rarity of PEL, few studies have examined its pathogenesis and genetic features. There are no clear identifiable risk factors. However, rare cases of $d e$ novo familial PEL, being autosomal dominant, have been reported. ${ }^{9,10} \mathrm{~A}$ number of available studies have revealed complex karyotype with predominance of $-5 / \operatorname{del}(5 q),-7 / \operatorname{del}(7 q)$ and +19 p. ${ }^{1,7,8,11-13}$ In a study conducted by Liu $e t$ al., all PEL patients $(n=16)$ showed an extremely complex karyotype, with a median of 12 abnormalities (range 3-37). These abnormalities most frequently involved chromosomes 5 (69\%), 7 (56\%), 17 (44\%), and 19 (38\%). ${ }^{1}$

Cytogenetic study for the present patient revealed an extreme complex karyotype with 8 chromosomal abnormalities. Beside the most commonly reported abnormalities, such as $\operatorname{del}(5 q)$ and $\operatorname{del}(7 q)$, our patient had a novel translocation $\mathrm{t}(8 ; 9)(\mathrm{p} 11.2 ; \mathrm{q} 12)$ in all the examined metaphases. To the best of our knowledge, this translocation has never been reported in acute myeloid leukemia in the past. Unfortunately, we were not able to perform a higher molecular study in order to clarify the origin of this abnormality.

Diagnosis of PEL requires multiparameter approach including detailed clinical history, peripheral blood and bone marrow examination, immunophenotyping and genetic studies as well.

Peripheral blood smears reveal non specific findings in the majority of PEL cases. However, about one quarter of patients show circulating erythroblasts. ${ }^{1}$ Anemia and thrombocytopenia are frequently observed while neutrophil counts vary. ${ }^{2}$ The present case also showed pancytopenia with $3 \%$ circulating blasts in the peripheral blood.

In bone marrow aspirate, the majority of cells $(>80 \%)$ are erythroid precursors with marked left shift and increased proerythroblasts. Proerythroblasts-the most immature recognizable erythroid precursors- are medium to large blasts with round nuclei, fine chromatin, occasional prominent nucleoli, deep blue

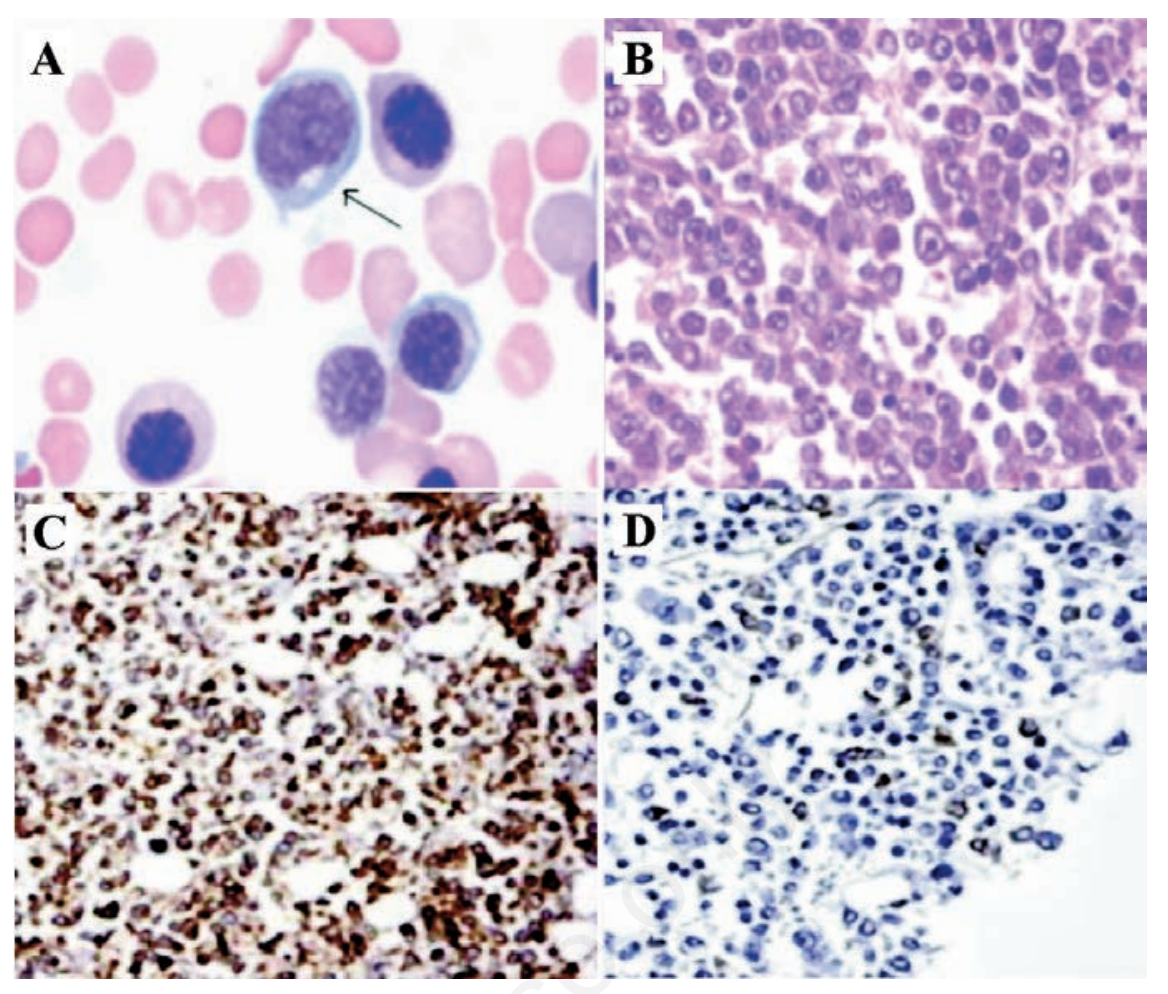

Figure 1. Morphological and Immunohistochemistry findings of bone marrow. A) Bone marrow aspirate with erythroid precursors and many proerythroblasts (arrow). The erythroblasts are medium with scant cytoplasm, high nuclear to cytoplasmic ratio, round nuclei with fine chromatins and 1-2 prominent nucleoli. B) Bone marrow biopsy is hypercellular and packed with monotonous leukemic infiltrate composed of primitive looking blasts. (H\&E, $\times 100) . C)$ Proerythroblasts are strongly positive for E-cadherin. D) Glycophorin A shows weak positivity.

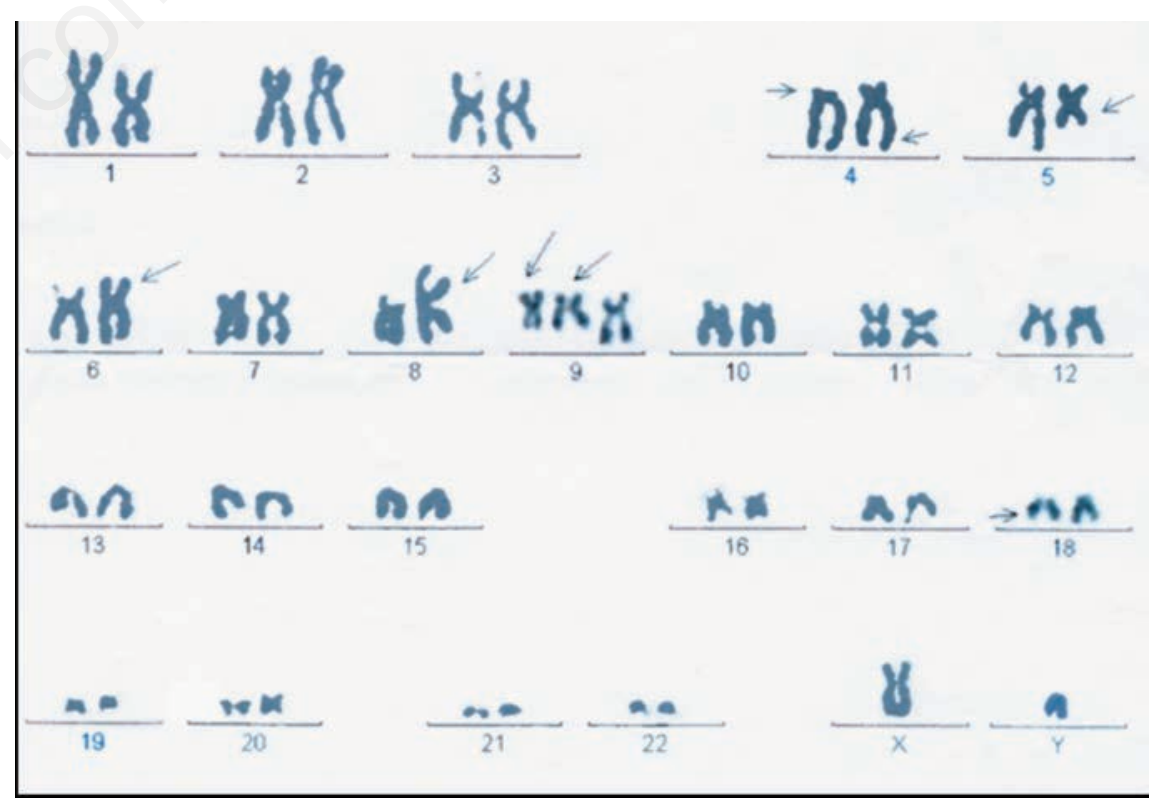

Figure 2. Complex karyotype with multiple chromosomal aberrations: 46-48,XY, $\operatorname{add}(4)(q ?), \operatorname{del}(4)(p ?), \operatorname{del}(5)(q 23), \operatorname{add}(6)(p 23), \operatorname{del}(7)(q 22), \mathbf{t}(8 ; 9)(\mathrm{p} 11.2 ; q 12)$, $+\operatorname{del}(9)($ q22), $\operatorname{del}(18)($ q21). 
agranular cytoplasm with variable cytoplasmic vacuolization and no Auer rods. ${ }^{14}$ Dyserythropoiesis is prominent, while myeloid and megakaryocytic dysplasia is not an obvious feature. ${ }^{5}$

Periodic Acid-Schiff (PAS) cytochemical staining is commonly positive, often in cytoplasmic vacuoles in a block-like pattern, while MPO, Sudan Black, and chloroacetate esterase are characteristically negative. ${ }^{15}$ Core biopsy is usually markedly hypercellular and shows erythroid predominance with sheets of immature cells clearly demonstrating a leukemic infiltrative pattern. ${ }^{1}$

Erythroblasts usually express CD71 (transferrin receptor), but occasionally CD71 expression can be aberrantly dim. These cells variably express hemoglobin A, glycophorin A, ABH blood group antigens, and HLA-DR. Erythroblasts are usually negative for myeloidassociated markers such as MPO. The Gerbich blood group (Gero) antibody, carbonic anhydrase1, and CD36 can be expressed by more immature erythroblast. ${ }^{16-19}$

Immunophenotyping of the present case revealed many immature cells which were positive for CD71, glycophorin A and partially positive for CD36, consistent with erythroid precursors. Myeloblasts were not detected.

Various markers including glycophorin A, B, and $\mathrm{C}$, hemoglobin, CD36, and spectrin have been used to identify immature erythroblasts in PEL; however, the results either lack consistency or specificity. ${ }^{20,21}$

E-cadherin is a cell adhesion molecule involved in the morphogenesis of multicellular organisms and maintenance of solid tissues. It is a selective marker of immature erythroblasts and is down regulated during erythroid maturation. In the bone marrow, its expression appears to be limited to erythroid lineage, and mainly in immature erythroblasts. ${ }^{22-24}$

In the present patient, e-cadherin immunohistochemistry stain revealed strong positivity $(\approx 80 \%)$ while glycophorine glycophorin A was weak positive $(\approx 20)$ which was in agreement with the published data. The combination of Ecadherin with glycophorin A effectively distinguishes immature and mature erythroid precursors.

Differential diagnosis of PEL is broad, and includes both non-neoplastic and neoplastic conditions. Erythroid hyperplasia may result from ineffective erythropoiesis such as congenital dyserythropoietic anemia, hemolytic anemia, vitamin B12/folate deficiency, infection, or autoimmune hemolysis. Importantly, previous erythropoietin treatment can cause reactive erythropoiesis that mimics pure erythroid leukemia. ${ }^{25}$ Even marked dyserythropoiesis can be seen in floridly reactive conditions potentially mimicking a myeloid neoplasm. $^{5}$

Pure erythroid leukemia needs also, to be distinguished from other forms of myeloid neoplasms with erythroid predominance namely therapy related ( $\mathrm{t}$-AML), acute myeloid leukemia with myelodysplasia related changes (AML-MRC), AML with recurrent cytogenetic abnormalities and myelodysplastic syndrome. Cases that do not fit into any of the above welldefined categories are classified as AMLNOS, further subcategorized by their morphologic and immunophenotypic characteristics. Thus, PEL is largely a diagnosis of exclusion. ${ }^{2}$ Pure erythroid leukemia without morphologic evidence of erythroid maturation may be difficult to be distinguished from other types of AML, particularly megakaryoblastic leukemia, and also from acute lymphoblastic leukemia and lymphoma.

\section{Treatment and prognosis}

Pure erythroid leukemia is a very aggressive disease with dismal prognosis and poor outcome. The median survival is 3.6 months compared with 28 months in acute erythroid/myeloid leukemia. ${ }^{1,5}$ The standard induction for PEL is $(7+3)$ chemotherapy regimen, consisting of daunorubicin $45 \mathrm{mg} / \mathrm{m}^{2}$ daily for 3 days and cytarabine $100 \mathrm{mg} / \mathrm{m}^{2}$ daily continuous infusion for 7 days; which offers a complete remission (CR) rate of approximately $10-40 \%$; if CR is obtained, it is usually brief.

The response to chemotherapy and the length of survival is dependent on several factors such as cytogenetics and karyotype abnormality and patient comobidities. ${ }^{14,15-17}$

\section{References}

1. Liu W, Hasserjian RP, Hu Y, et al. Pure erythroid leukemia: a reassessment of the entity using the 2008 World Health Organization classification. Mod Pathol 2011;24:375-83.

2. Arber DA, Brunning RD, Orazi A, et al. Acute myeloid leukemia, not otherwise specified. In: Swerdlow SH, Campo E, Harris NL, et al, eds. Tumours of haematopoietic and lymphoid tissues. Lyon: IARC Press; 2008. pp 134-136.

3. Olopade OI, Thangavelu M, Larson RA, et al. Clinical, morphologic, and cytogenetic characteristics of 26 patients with acute erythroblastic leukemia. Blood 1992;80: 2873-82.

4. Colita A, Belhabri A, Chelghoum Y, et al. Prognostic factors and treatment effects on survival in acute myeloid leukemia of M6 subtype: a retrospective study of 54 cases. Ann Oncol 2001;12:451-5.

5. Mihova D, Zhang L. Acute erythroid leukemia: a review. N A J Med Sci 2012;5:110-8.

6. Keifer J, Zaino R, Ballard J0.
Erythroleukemic infiltration of a lymph node: use of hemoglobin immunohistochemical techniques in diagnosis. Hum Pathol 1984;15:1090-3.

7. Latif N, Salazar E, Khan R, et at. The pure erythroleukemia: a case report and literature review. Clin Adv Hematol Oncol 2010;4:283-8.

8. Suresh PK, Rao PS, Khalidkar UN. Erythroleukemia with complex cytogenetic abnormalities: a case report with review of literature. Indian J Appl Res 2013:7;539-40.

9. Haught EAS, Johnson MC, Witt PD. Congenital erythroleukemia presenting as a congenital infantile hemangioma. Plast Reconstr Surg 2007;119:e70-2.

10. Novik Y, Marino P, Makower DF, Wiernik PH. Familial erythroleukemia: a distinct clinical and genetic type of familial leukemias. Leuk Lymphoma 1998;30:395401.

11. Bacher U, Haferlach C, Kern W, et al. Prognostic relevance of FLT3-TKD mutations in AML: the combination matters: an analysis of 3082 patients. Blood 2008;111:2527-37.

12. Marcucci G, Maharry K, Whitman SP, et al. High expression levels of the ETS-related gene, ERG, predict adverse outcome and improve molecular risk based classification of cytogenetically normal acute myeloid leukemia: a Cancer and Leukemia Group B study. J Clin Oncol 2007;25:333743.

13. Acquaviva C, Gelsi-Boyer V, Birnbaum D. Myelodysplastic syndromes: lost between two states? Leukemia 2010;24:1-5.

14. Hasserjian RP, Zuo Z, Garcia C, et al. Acute erythroid leukemia: a reassessment using criteria refined in the 2008 WHO classification. Blood 2010;115:1985-92.

15. Zuo Z, Polski JM, Kasyan A, et el. Acute erythroid leukemia. Arch Pathol Lab Med 2010;134:1261-70.

16. Bain BJ. Di Guglielmo and his syndromes. Br J Haematol 2003;120:939-43.

17. Lowenberg B, Downing JR, Burnett A. Acute myeloid leukemia. N Engl J Med 1999;341:1051-62.

18. Kogawa K, Sekine I, Masuda T, et al. Bone marrow transplantation for erythroleukemia: a case report. Acta Paediatr Jpn 1994;36:693-6.

19. Killick S, Matutes E, Powles RL, et al. Acute erythroid leukemia (M6): outcome of bone marrow transplantation. Leuk Lymphoma 1999;35:99-107.

20. Garand R, Duchayne E, Blanchard D, et al. Minimally differentiated erythroleukaemia (AML M6 variant): a rare subset of AML distinct from AML M6. Groupe Francais d'Hematologie Cellulaire. Br J Haematol 1995;90:868-75.

21. Sadahira Y, Kanzaki A, Wada H, et al. 
Immunohistochemical identification of erythroid precursors in paraffin embedded bone marrow sections: spectrin is a superior marker to glycophorin. J Clin Pathol 1999;52:919-21.

22. Acs G, LiVolsi VA. Loss of membrane expression of E-cadherin in leukemic erythroblasts. Arch Pathol Lab Med
2001;125:198-201.

23. Armeanu S, Buhring HJ, Reuss-Borst M, et al. E-cadherin is functionally involved in the maturation of the erythroid lineage. $\mathrm{J}$ Cell Biol 1995;131:243-9.

24. Buhring HJ, Muller T, Herbst R, et al. The adhesion molecule E-cadherin and a surface antigen recognized by the antibody
9C4 are selectively expressed on erythroid cells of defined maturational stages. Leukemia 1996;10:106-16.

25. Moharram L, Kamal N, Sukhun SA, Sughayer MA. Erythropoietin-induced acute erythroid leukemia-like picture: a potential pitfall. Hematol Oncol Stem Cell Ther 2014;7:50-2. 